Methods Three PPM simulations were carried out in a general paediatric setting. Themes: acute presentation of a patient with no treatment ceiling decisions in place but parents not wishing for escalation of treatment; putting an advance care plan/DNACPR into place during acute presentation of a dying child and managing an expected death. Simulations were attended by medical and nursing staff with 2-3 staff actively participating and others observing. Two simulations were run with a high-fidelity mannequin and one with a low fidelity mannequin. Supporting items were used including DNACPR and EHCP (emergency health care plan) documentation, clinic letters/ward round entries and drug prescriptions.

All participants were asked to complete feedback at the end of each session and active participants were contacted following the session for further feedback. A five point Likert scale was used to rate enjoyment, organisation, quality and relevance of session and likelihood of attending further simulation teaching in PPM. A 10-point confidence scale was used to rate confidence in dealing with PPM scenarios and confidence in talking to families about death both before and after the scenario. Additional comments were also collected.

Ethical approval was not required.

Results Participants reported they enjoyed the sessions (mean score $4.7 / 5$; range $0-5$ ); felt they were well organised $(4.7 / 5 ; 1-5)$ and of high quality $(4.7 / 5 ; 1-5)$. They felt sessions were relevant to their training $(4.5 / 5 ; 1-5)$ and that they would attend further simulation training in the area of PPM (mean 4.8/5; range 1-5). Confidence in managing PPM scenarios increased by a mean of 2.4 confidence points (range $0-5$ ) and confidence in managing conversations around death increased 1.8 confidence points (range 0-5).

Conclusion Simulation training to teach PPM skills to the multi-disciplinary paediatric team was acceptable to participants and improved participants' confidence in managing PPM scenarios and conversations around death.

\section{G363(P) SHARE OUR LEARNING EXPERIENCE AND APPROACH USED TO DEAL WITH A CHALLENGING COMPLEX CASE INCLUDING SAFEGUARDING CONCERNS AND ETHICAL ISSUES IN A COMMUNITY SETTING}

${ }^{1} \mathrm{R}$ Jainer, ${ }^{1,2}$ E Isles. 'Department of Paediatrics, University Hospitals of Birmingham NHS Trust, Solihull, UK; ${ }^{2}$ Community Nursing Team, University Hospitals of Birmingham NHS Trust, Solihull, UK

\subsection{6/archdischild-2020-rcpch.311}

Aims Share our learning experience of dealing with a challenging complex case with life limiting condition called Canavans disease in a Community setting.

It was an acute life threatening safeguarding concern on background of long standing concerns by health professional on a 17 year old. He had complex health care needs including respiratory support and gastrostomy feeds. He is dependent on adults for all his care and administration of medications. He was on child in need plan due to long standing concerns.

Family suddenly expressed a wish to take him on a long haul flight to attend a family wedding to meet their own emotional and social needs.
Method Information gathering from varied professionals. Multiagency meetings and sharing information transparently.

Presentation at Regional palliative care meeting, no consensus reached. Risks assessment completed and discussed with family about safety, dignity, pain relief and risk of deterioration on flight and empathised that despite efforts to mitigate concerns would not be in his best interest.

Family was not in agreement with decision of professionals

Escalated concerns with safeguarding lead and advice from legal team taken.

Best interest team meeting was arranged.

Result Decision in best interest of the young person not to fly as risks outweighed the benefits.

Young person was abandoned by family without appropriate carer to attend to his needs. He was then made ward of court and admitted in hospital till residential placement was arranged and transited to adult services.

Conclusion Despite adherence to principles of palliative care which was holistic, patient centred care and transition to adult services in a community setting, we are faced with ethical and legal challenges.

However, challenge was dealt with clinical leadership and team, effective communication skills and peer support and all having a common goal of compassion and ensuring safety of the young person.

This also prevented an impending safeguarding practice review

Recommendations Sharing of more such cases in a wider forum to develop better understanding for clinicians about what avenues are accessible. Having training days jointly organised by Paediatric palliative care with input from safeguarding leads would help clinicians acquire the needed skills for dealing with such complex ethical cases.

\section{G364(P) WHAT SIGNS OR SYMPTOMS INDICATE A CHILD IS LIKELY TO DIE WITHIN MONTHS OR DAYS AND ARE THEY BEING USED WITHIN THIS HOSPITAL?}

${ }^{1} S$ Lovegrove, ${ }^{2,3} \mathrm{~J}$ Manning, ${ }^{2} \mathrm{~T}$ Wolff. ${ }^{1}$ School of Medicine, University of Nottingham, Nottingham, UK; ${ }^{2}$ Nottingham Children's Hospital, Nottingham University Hospitals, Nottingham, UK; ${ }^{3}$ School of Health Sciences, University of Nottingham, Nottingham, UK

10.1136/archdischild-2020-rcpch.312

Background Clinicians must be able to spot when children with life-limiting and life- threatening conditions might be approaching the last 6-12 months of life, to allow time for advance care planning to be put in place. As yet, no prognostic indicator tool exists for paediatric palliative care. The Chameleon children's end of life (EoL) care quality improvement project promoted the use of an EoL care prompt during 2018. The purpose of this audit was to then determine if the addition of prognostic indicators to the prompt tool may improve its use and thus the timely implementation of essential interventions.

Objectives To determine locally used prognostic indicators for the last 6-12 months of a child's life within this Children's Hospital. To retrospectively ascertain if they have been used and if appropriate interventions have been completed from the EoL care prompt.

Methods 12 healthcare professionals were interviewed to find locally used prognostic indicators. Children who died an expected death as a result of either an oncological or 